\title{
Monte Carlo simulation of near-field terahertz emission from semiconductors
}

\author{
S. C. Corzo-Garcia, A. I. Hernandez-Serrano, and E. Castro-Camus* \\ Centro de Investigaciones en Optica A.C., Loma del Bosque 115, Lomas del Campestre, Leon, Guanajuato 37150, Mexico \\ O. Mitrofanov \\ Department of Electronic and Electrical Engineering, University College London, London WC1E 7JE, United Kingdom
}

(Received 5 April 2016; published 1 July 2016)

\begin{abstract}
We simulated the carrier dynamics in InGaAs after ultrafast photoexcitation. By using a finite-difference time-domain approach we were able to analyze the near terahertz field emission caused by the motion of such carriers. We found that both the current parallel and normal to the interface take a relevant role in the terahertz emission. We also found that the ballistic motion of the carriers after photoexcitation dominates the emission rather than diffusion.
\end{abstract}

DOI: 10.1103/PhysRevB.94.045301

\section{INTRODUCTION}

The effect of emission of single-cycle subpicosecond electromagnetic pulses upon ultrashort optical excitation of a semiconductor surface $[1,2]$ found widespread application in terahertz $(\mathrm{THz})$ time-domain spectroscopy. The underlying mechanisms, however, are still under investigation. For the optical excitation with photon energies above the band gap, the electromagnetic pulses with spectral components across the terahertz band are produced predominantly by a surge of photocurrent [2-4]. The direction of the photocurrent vector in unbiased semiconductors has been identified as perpendicular to the surface, usually driven by the built-in surface field of the material [2,5-7]. In semiconductors with small surface built-in fields, the photocurrent was associated with the photo-Dember effect $[8,9]$. Photocurrents in the direction parallel to the surface have been considered negligible until only recently, when several experimental studies reported emission of $\mathrm{THz}$ pulses produced by the carrier motion in the surface plane [10-14]. In particular, $\mathrm{THz}$ radiation was observed upon asymmetric photoexcitation of the semiconductor surface, e.g., by illuminating the surface near an edge of a metallic film or by clipping the excitation beam on one side [12,13]. More recently, near-field mapping of the $\mathrm{THz}$ field generated by InGaAs surfaces illuminated at normal incidence showed that the source of $\mathrm{THz}$ radiation is not located in the center of photoexcitation [15]. Instead, regions where the gradient of the in-plane photocarrier density is highest, i.e., the edges of the photoexcited region, were found to radiate the strongest $\mathrm{THz}$ field [15]. This counterintuitive fact suggests that unbalanced (ambipolar) expansion of the electron-hole ensembles within the surface plays an important role in the process of $\mathrm{THz}$ pulse generation. Furthermore, the amplitude of the field radiated from the edges of the normal incidence excitation was found to be comparable to the amplitude of the field radiated by the same surface excited at $45^{\circ}$ incidence, known to be optimal for $\mathrm{THz}$ pulse emission. It suggests that the contribution of the in-plane transient currents is underestimated [15].

Despite the growing experimental evidence that photocurrents in the surface plane contribute to the radiated $\mathrm{THz}$

\footnotetext{
*enrique@cio.mx
}

field, none of the recent studies have definitively addressed the essential question: which of the photocurrents, normal or parallel to the surface, emits $\mathrm{THz}$ pulses most efficiently? The driving force in the direction normal to the surface, i.e., the built-in field or the carrier concentration gradient, is typically stronger, producing higher photocurrents in that direction. On the other hand, photocurrents in the surface plane can be coupled out of the material and into free-space more efficiently.

In order to quantify contributions of the two orthogonal photocurrent components, normal and parallel to the surface, we model the photocarrier dynamics produced by the ultrashort optical excitation using Monte Carlo simulations. The spaceand time-dependent photocurrent is then used to compute the radiated $\mathrm{THz}$ field distribution, which is compared to the experimentally observed distribution to verify the validity of the simulations. We find excellent agreement between the experiment and the simulations. The simulated carrier dynamics are then used to separate the $\mathrm{THz}$ field radiated by each one of the two orthogonal photocurrent components. This analysis allows us to conclude that the photocurrent transients in the direction normal to the surface and parallel to the surface radiate $\mathrm{THz}$ pulses of comparable amplitude. We find that the low $(<300 \mathrm{GHz})$ frequency components of the radiated spectrum are caused by the initial transient current, while the high $(>1.5 \mathrm{THz})$ frequencies are produced by the plasma oscillations in the direction normal to the surface.

\section{MODEL}

The semiclassical Monte Carlo simulation of charge carrier dynamics used in this investigation works as follows. Ten million pseudoparticles representing charge carriers are assumed to move classically in a three-dimensional space, following Newton's second law, over 1 fs time periods. Initially a random distribution of intrinsic carriers with energies given by a Maxwell-Boltzmann distribution is assumed. The charge density is calculated on a grid dividing the space, from it, the Poisson equation is solved and the electric field is calculated. For each time interval, quantum mechanical scattering rates are calculated and pseudorandom numbers are used to decide if the carriers scatter, as well as to determine their scattering angle and energy loss. The carrier scattering mechanisms we assumed are LO phonons, TO-phonon mediated intervalley 

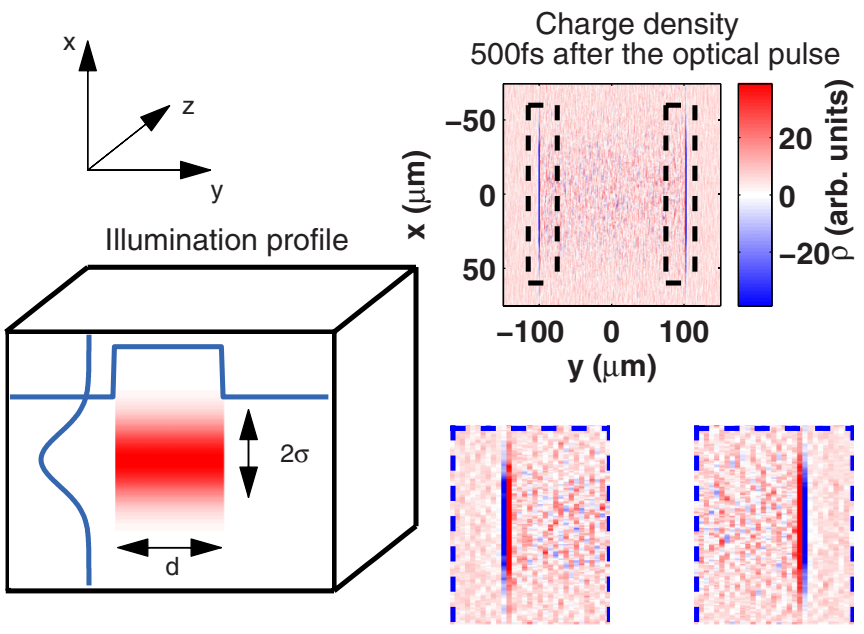

FIG. 1. Schematically shows the intensity pattern used (left): it follows a Gaussian profile in the $x$ direction, while it is a step function in the $y$ direction indicated by the blue lines. The charge density from the simulation $500 \mathrm{fs}$ after photoexcitation is also shown (right). Dashed lines indicate regions that are shown in greater detail in the two smaller panels below.

( $\Gamma, L$, and $X)$, acoustic phonons, charged impurity, and carrier carrier. This process is repeated to simulate the carrier motion for the duration of $8 \mathrm{ps}$. Further details on the simulation can be found in Refs. [16-18].

In order to enable comparison with the $\mathrm{THz}$ field distribution observed in experiments, the photocarriers were injected into the simulation space using a Gaussian distribution in the $x$ direction $(\sigma=30 \mu \mathrm{m})$, a steplike distribution in the $y$ direction $(d=200 \mu \mathrm{m})$, and an exponential distribution in the $z$ direction as schematically depicted in Fig. 1. The optical pulse responsible for photoinjection was assumed to have a Gaussian photon energy distribution consistent with a $50 \mathrm{fs}$ pulse centered at $800 \mathrm{~nm}$. Each one of the photoinjected electron-hole pairs in the simulation was assumed to have an energy equal to the difference between each photon and the semiconductor's band gap. The energy was distributed between the two carriers imposing momentum conservation in a random direction across $4 \pi$. All simulations assumed a temperature of $300 \mathrm{~K}$.

We used the two-dimensional transverse cut on the $y z$ plane $(x=0)$, space- and time-dependent charge density in order to calculate the local current density for each time step. This current density was then used as the source term in Maxwell's "curl" equations

$$
\nabla \times \mathbf{E}_{\mathrm{THz}}=-\frac{\partial \mathbf{B}_{\mathrm{THz}}}{\partial t}
$$

and

$$
\nabla \times \mathbf{B}_{\mathrm{THz}}=\frac{1}{c^{2}} \frac{\partial \mathbf{E}_{\mathrm{THz}}}{\partial t}+\mu_{0} \mathbf{J},
$$

which were subsequently solved numerically in a finitedifference time-domain fashion on the $y z$ plane in order to obtain the emitted terahertz fields $\mathbf{E}_{\mathrm{THz}}$ and $\mathbf{B}_{\mathrm{THz}}$ [19]. This model provides a very detailed picture of both the carrier dynamics and electromagnetic aspects of the near-field terahertz emission process from photoexcited semiconductors.
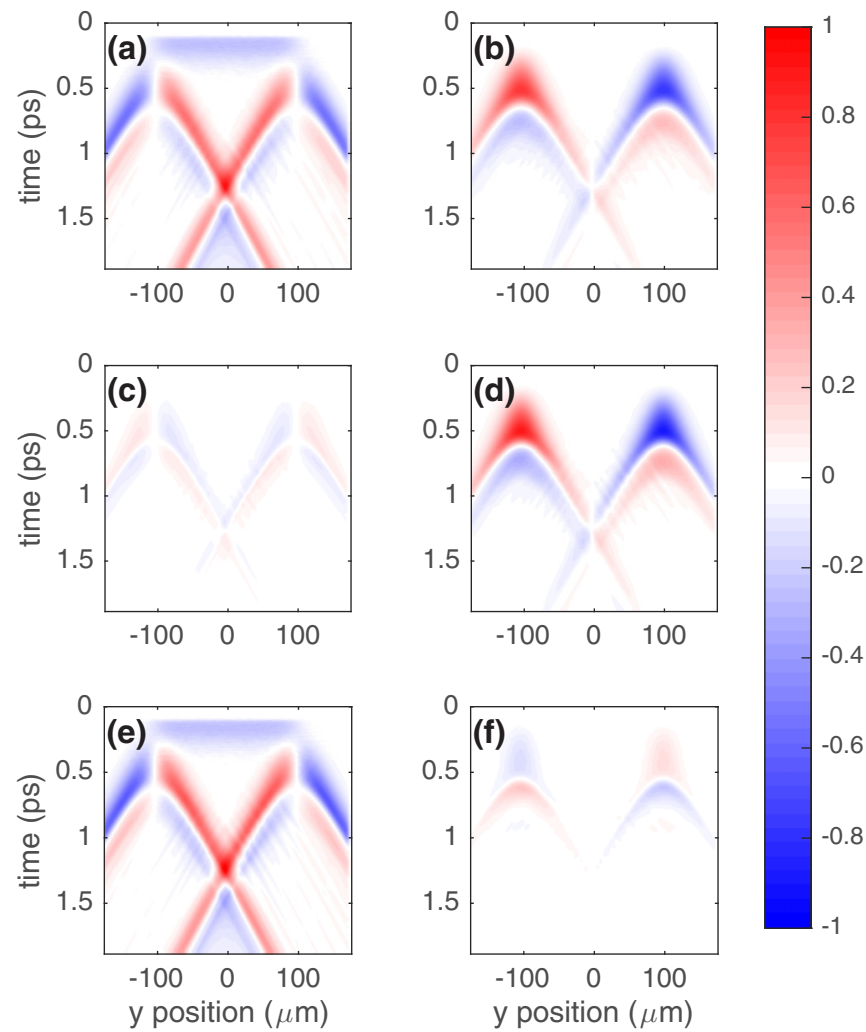

FIG. 2. Electric fields from the Monte Carlo simulation in the near field as function of the position and time. (a) The $E_{z}$ component produced by $\mathbf{J}$, (b) the $E_{y}$ produced by $\mathbf{J}$, (c) the $E_{z}$ component produced only by $J_{y}$, (d) the $E_{y}$ component produced by $J_{y}$, (e) the $E_{z}$ component produced only by $J_{z}$, and (f) the $E_{y}$ component produced only by the $J_{z}$ component.

\section{RESULTS}

Before starting the discussion of the results, it is worth mentioning that no fitting parameters were used in order to reproduce the experimental results. All the parameters used in the carrier dynamics simulation correspond to the known parameters of InGaAs and were taken from Refs. [16,18]. Furthermore the arbitrary units used in all the simulated electric field plots are consistent with each other (i.e., they are the same arbitrary units). Likewise, the signal arbitrary units are also consistent with each other.

Figure 2 shows the generated $\mathrm{THz}$ field in the forward direction at the distance of $30 \mu \mathrm{m}$ from the surface. The right column panels show the $E_{y}$ component and the left column show the $E_{z}$ component. The top row [Figs. 2(a) and 2(b)] show the electric fields produced by the full current vector $\mathbf{J}$. The middle row [Figs. 2(c) and 2(d)] show the radiated field produced only by the current parallel to the surface $\left(J_{y}\right)$ and the panels in the lower row are the electric field components produced only by the current normal to the surface $\left(J_{z}\right)$.

As expected, the electric field with polarization parallel to the surface is produced mainly by the current parallel to the surface [Fig. 2(d)], and the electric field with polarization normal to the surface is produced mainly by the current normal to the surface too [Fig. 2(e)]. Both currents produce $\mathrm{THz}$ fields with comparable amplitudes. There is also a relatively 

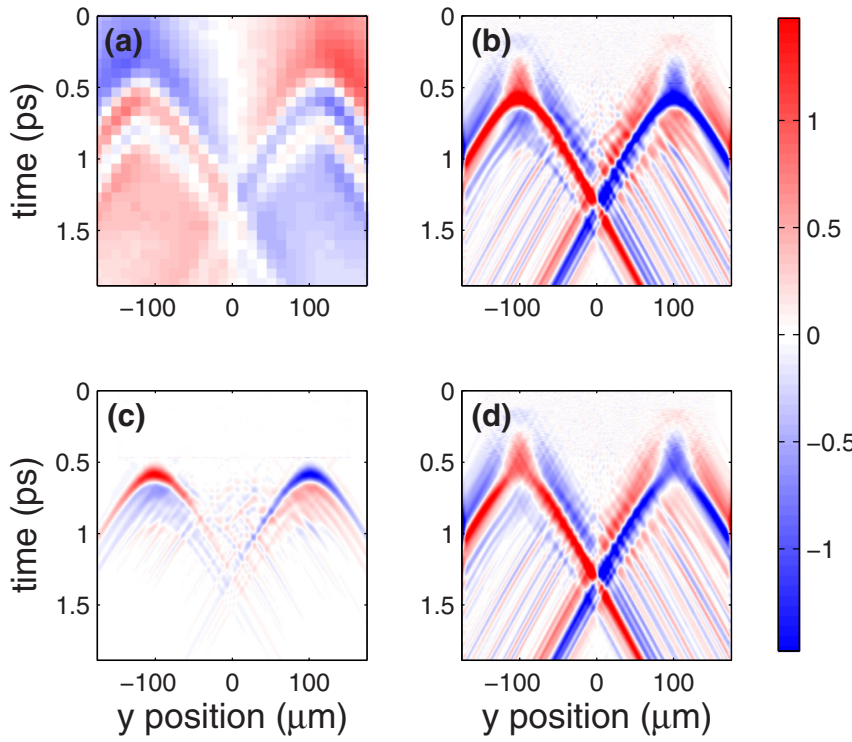

FIG. 3. (a) Electric field as function of position and time obtained from the experiment. (b) Theoretically calculated signal. Panels (c) and (d) are the theoretically calculated contributions to the signal form for the $J_{y}$ and $J_{z}$ components of the current, respectively.

small but nonzero contribution of the crossed terms [Figs. 2(c) and 2(f)].

In order to validate the simulation results we compare them to the electric field distribution reported in experiments in Ref. [15]. It must be noted that the near-field probe is sensitive to the two vector components of the $\mathrm{THz}$ field [20]. It was shown experimentally that, in the case of the $E_{y}$ component, the probe detects the temporal derivative of the incident field $E_{y}$ [21], whereas in the case of the $E_{z}$ component, it detects a spatial derivative of the field [22]. The probe sensitivity to the $E_{y}$ and $E_{z}$ components of the field has been verified numerically [22]. We can estimate the detected near-field signal directly from the simulated $E_{y}$ and $E_{z}$ fields using the following expressions [23]:

$$
\begin{aligned}
S_{y}(t, y) & \propto a \frac{n}{c} \frac{\partial E y(t, y)}{\partial t}, \\
S_{z}(t, y) & \propto a \frac{\partial E z(t, y)}{\partial y},
\end{aligned}
$$

where $a$ is the aperture size and $c$ is the speed of light.

Figures 3(a) and 3(b) show the radiated field from the experiment and the combined signal $\left(S_{y}+S_{z}\right)$ as functions of the probe position respectively for InGaAs illuminated by a $200 \mu \mathrm{m}$ wide strip of light at normal incidence. The numerically simulated map [Fig. 3(b)] of the detected signal is in remarkable agreement with the experimental map shown in Fig. 3(a). Figures 3(c) and 3(d) show the two signal components separately. The maps of the detected field $S_{y}$ and $S_{z}$ are similar; however, there are distinctive differences: the detected signal due to $S_{z}$ field has longer "tails" and the field varies slower in time compared to the $S_{y}$.

In order to analyze the two components further, we plot the wave forms of the simulated signal contributions of $J_{y}\left(S_{y}\right)$ and $J_{z}\left(S_{z}\right)$ near the edge of the optical excitation, i.e., for the probe
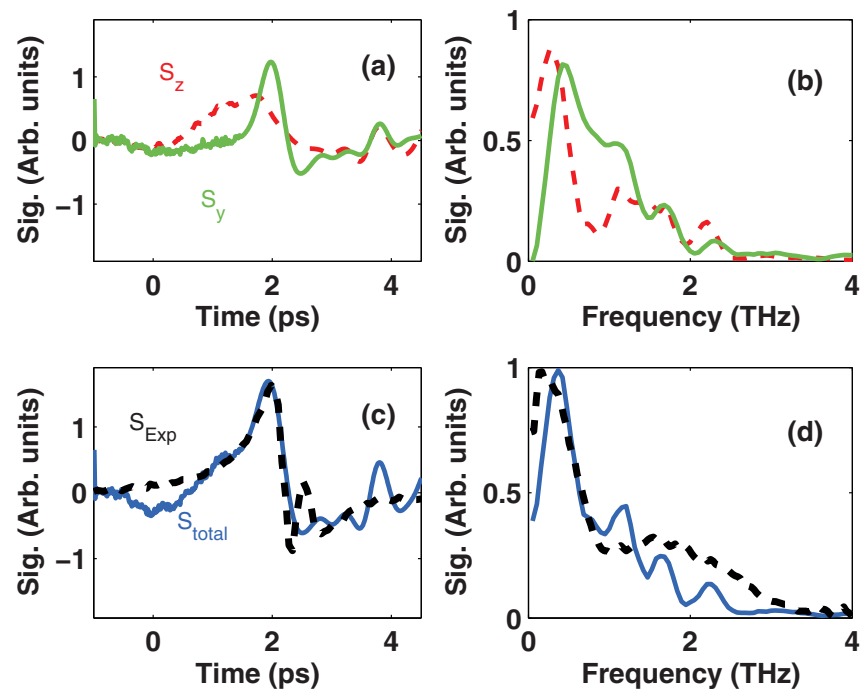

FIG. 4. (a) Continuous curve is the calculated time-domain wave form at the position of the illumination edge. The dashed and continuous lines are the contributions to the signal from $J_{z}$ and $J_{y}$, respectively. (b) The corresponding spectra for the wave forms shown in (a) following the same line-type convention. (c) The continuous line is the predicted signal produced by the full $\mathbf{J}$ vector and the dashed line is the experimental wave form. (d) The corresponding spectra of both the simulation for $\mathbf{J}$ and the experimental measurement.

location where the detected field is strongest (Fig. 4). The wave forms show more clearly that the $S_{z}$ component varies in time slower compared to $S_{y}$. The corresponding spectral peaks are therefore at different frequencies: $0.26 \mathrm{THz}$ for $S_{z}$ and $0.38 \mathrm{THz}$ for $S_{y}$. The amplitude of the radiated field due to the current normal to the surface exhibits a relatively quick drop in the $0.5 \mathrm{THz}$ region, reaching a minimum in the $0.9 \mathrm{THz}$ region. This component however recovers across the $1.0 \mathrm{THz}$ to $2.0 \mathrm{THz}$ band where it drops again.

The signal due to the in-plane current, $S_{y}$, on the other hand has a negligible contribution at the low frequencies, below $0.2 \mathrm{THz}$, where the $S_{z}$ components dominate. Yet, in the intermediate frequencies, between 0.6 and $1.5 \mathrm{THz}$, $S_{y}$ becomes the dominant contribution. It drops in power becoming again smaller than the $S_{z}$ component in the 2.0 to $4 \mathrm{THz}$ region. Both signals eventually disappear at around $2.5 \mathrm{THz}$.

Figure 4(c) compares the wave form predicted by our simulation, which accounts for the full $\mathbf{J}$ distribution, with the experimental wave form (dashed line). The duration and general form of the pulses, as well as their spectra, are similar. In particular, the two spectra [Fig. 4(d)] show their maxima in the $300 \mathrm{GHz}$ region followed by a fast drop in the $500 \mathrm{GHz}$ region, reaching about $28 \%$ of their maximum amplitude at $1 \mathrm{THz}$. In the region between $1 \mathrm{THz}$ and $2 \mathrm{THz}$, both spectra show an increase in the emitted energy. However, the simulated spectrum shows clear ripples, whereas the experimental spectrum is smooth.

The results presented in Fig. 4 allow us to draw the following conclusions: the dominant contribution to the low $(<300 \mathrm{GHz})$ and high $(>1.5 \mathrm{THz})$ frequency parts of the signals spectrum comes from the current normal to the surface 

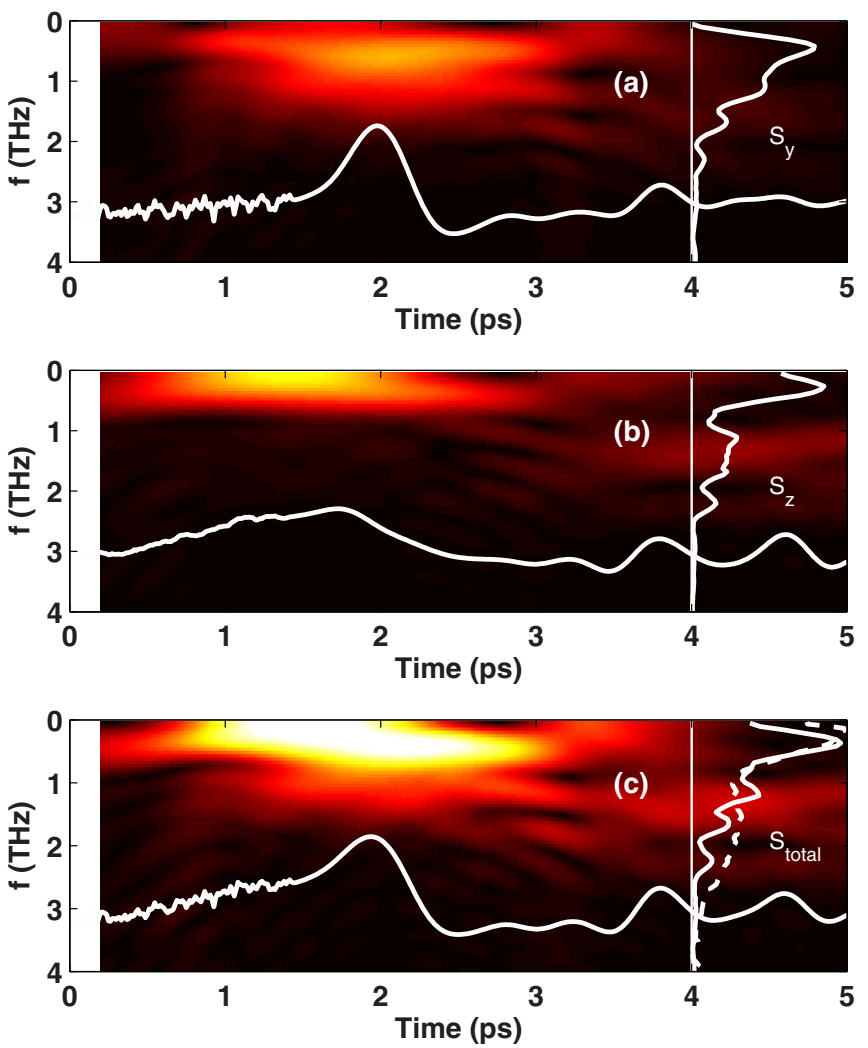

FIG. 5. Color maps represent the temporal evolution of the spectrum for the $S_{y}$ (a), $S_{z}$ (b), and $S_{\text {total }}$ (c) wave forms. The white curves shown on the bottom are the corresponding wave forms, and the curves on the right hand side are the spectrum of the full wave form provided as a reference for each panel. An additional dashed line is provided in panel (c) which corresponds to the experimental spectrum.

$\left(J_{z}\right)$, while in the intermediate region, between $600 \mathrm{GHz}$ and $1.5 \mathrm{THz}$, the contribution of the current parallel to the surface $\left(J_{y}\right)$ dominates.

The three color maps in Fig. 5 are spectrograms that show the evolution of the spectrum of the wave forms over time for the $y$ (a) and $z$ (b) components as well as the full current $\mathbf{J}$ (c). This calculation was performed by applying a 0.8 ps FWHM Gaussian filter to the wave forms and producing their Fourier transforms as the Gaussian moves in time. A careful analysis of the evolution of the spectrum shows that, while the main peak of the spectrum below $1 \mathrm{THz}$ is caused by the initial transient acceleration of carriers, in both the $y$ and $z$ directions, the high frequency components producing the moderate increase in power between $1 \mathrm{THz}$ and $4 \mathrm{THz}$ is produced by more subtle oscillations that are distributed from the moment of the excitation $(t=0)$ onwards.
While the initial transient is, at least qualitatively, not very dependent on the precise conditions of the carrier dynamics, the smaller oscillations are more susceptible to the details of the carrier motion conditions, in particular, the carrier density. As described earlier, the electromagnetic calculations are performed considering only the two-dimensional slice at the center of the space simulated by the Monte Carlo code. Therefore, the calculations presented correspond to a specific carrier density. The measurement however incorporates the contribution of radiation emitted at different $x$ positions, which have different carrier densities. This explains why the experiment shows a broad continuous distribution between $1 \mathrm{THz}$ and $2.5 \mathrm{THz}$. Simulations for various values of the injected carrier densities were performed in order to confirm this.

Further simulations were performed without the carriercarrier scattering. Only marginal differences could be seen in the spectra, suggesting that the main contribution to the dipole formation, and therefore to the $\mathrm{THz}$ emission, is from the initial photoinjection velocity of the carriers and from further ballistic transport rather than from diffusion. It is worth mentioning that our carrier dynamics simulation does not include any anisotropies related to the material which is appropriate for photoinjection at normal incidence on a [100] zinc-blende surface such as the InGaAs used in the experiments. Additional experimental measurements with the InGaAs sample at various angles around the [100] axis were performed, no significant differences were observed that could be associated to any material anisotropy.

\section{CONCLUSIONS}

The Monte Carlo simulations of the carrier dynamics combined with FDTD electromagnetic simulation of the radiated field reveal that the radiated field contains a relatively complex mix of contributions from the currents parallel and perpendicular to the semiconductor's surface. We find that the low $(<300 \mathrm{GHz})$ and high $(>1.5 \mathrm{THz})$ frequency components of the radiated spectrum are caused by the initial transient current and the plasma oscillations in the direction normal to the surface, respectively. The intermediate frequency components $(0.6-1.5 \mathrm{THz})$ have a more significant contribution from the current transient in the plane of the semiconductor surface. The simulated field agrees with the experimental results recorded recently using $\mathrm{THz}$ near-field microscopy. The numerical simulations show that an in-plane transient dipole moment forms at the edges of the optical excitation area immediately after the excitation. Correspondingly, the radiated field within the first $1-2$ ps after the excitation has a strong contribution from the in-plane carrier dynamics. As there is no driving field in the surface plane, the origin of this terahertz radiation is the transient dipole moment formed at the edges of the optical excitation area caused by different initial velocities of photoexcited electrons and holes.
[1] X.-C. Zhang, B. Hu, J. Darrow, and D. Auston, Appl. Phys. Lett. 56, 1011 (1990).

[2] X.-C. Zhang and D. Auston, J. Appl. Phys. 71, 326 (1992).
[3] J. Heyman, N. Coates, A. Reinhardt, and G. Strasser, Appl. Phys. Lett. 83, 5476 (2003).

[4] K. Liu, J. Xu, T. Yuan, and X.-C. Zhang, Phys. Rev. B 73, 155330 (2006). 
[5] H. G. Roskos, M. C. Nuss, J. Shah, K. Leo, D. A. B. Miller, A. M. Fox, S. Schmitt-Rink, and K. Köhler, Phys. Rev. Lett. 68, 2216 (1992).

[6] P. C. M. Planken, M. C. Nuss, I. Brener, K. W. Goossen, M. S. C. Luo, S. L. Chuang, and L. Pfeiffer, Phys. Rev. Lett. 69, 3800 (1992).

[7] J. N. Heyman, P. Neocleous, D. Hebert, P. A. Crowell, T. Müller, and K. Unterrainer, Phys. Rev. B 64, 085202 (2001).

[8] A. Reklaitis, J. Appl. Phys. 109, 083108 (2011).

[9] A. Reklaitis, J. Appl. Phys. 108, 053102 (2010).

[10] G. Klatt, F. Hilser, W. Qiao, M. Beck, R. Gebs, A. Bartels, K. Huska, U. Lemmer, G. Bastian, M. Johnston et al., Opt. Express 18, 4939 (2010).

[11] G. Klatt, B. Surrer, D. Stephan, O. Schubert, M. Fischer, J. Faist, A. Leitenstorfer, R. Huber, and T. Dekorsy, Appl. Phys. Lett. 98, 021114 (2011).

[12] M. Barnes, S. Berry, P. Gow, D. McBryde, G. Daniell, H. Beere, D. Ritchie, and V. Apostolopoulos, Opt. Express 21, 16263 (2013).

[13] M. Barnes, D. McBryde, G. Daniell, G. Whitworth, A. Chung, A. Quarterman, K. Wilcox, A. Brewer, H. Beere, D. Ritchie et al., Opt. Express 20, 8898 (2012).
[14] P. Cicenas, A. Geižutis, V. Malevich, and A. Krotkus, Opt. Lett. 40, 5164 (2015).

[15] R. Mueckstein, M. Natrella, O. Hatem, J. R. Freeman, C. S. Graham, C. C. Renaud, A. J. Seeds, E. H. Linfield, P. J. Cannard, M. J. Robertson et al., IEEE Trans. Terahertz Sci. Technol. 5, 260 (2015).

[16] M. B. Johnston, D. M. Whittaker, A. Corchia, A. G. Davies, and E. H. Linfield, Phys. Rev. B 65, 165301 (2002).

[17] E. Castro-Camus, J. Lloyd-Hughes, and M. B. Johnston, Phys. Rev. B 71, 195301 (2005).

[18] J. Lloyd-Hughes, E. Castro-Camus, and M. Johnston, Solid State Commun. 136, 595 (2005).

[19] C. Larsen, D. G. Cooke, and P. U. Jepsen, JOSA B 28, 1308 (2011).

[20] R. Mueckstein and O. Mitrofanov, Opt. Express 19, 3212 (2011).

[21] O. Mitrofanov, L. Pfeiffer, and K. West, Appl. Phys. Lett. 81, 1579 (2002).

[22] M. Natrella, O. Mitrofanov, R. Mueckstein, C. Graham, C. C. Renaud, and A. J. Seeds, Opt. Express 20, 16023 (2012).

[23] R. Mueckstein, C. Graham, C. C. Renaud, A. J. Seeds, J. A. Harrington, and O. Mitrofanov, J. Infrared, Millimeter, Terahertz Waves 32, 1031 (2011). 